\title{
New Theory of Effusive and Explosive Volcanic Eruptions
}

\author{
Alexander N. Safronov \\ Obukhov Institute of Atmospheric Physics, Russian Academy of Sciences/Pyzhevskii per. 3, Moscow, Russia \\ Email: safronov_2003@mail.ru
}

How to cite this paper: Safronov, A.N. (2022) New Theory of Effusive and Explosive Volcanic Eruptions. International Journal of Geosciences, 13, 115-137. https://doi.org/10.4236/ijg.2022.132007

Received: January 30, 2022

Accepted: February 25, 2022

Published: February 28, 2022

Copyright $\odot 2022$ by author(s) and Scientific Research Publishing Inc. This work is licensed under the Creative Commons Attribution International License (CC BY 4.0).

http://creativecommons.org/licenses/by/4.0/

\begin{abstract}
In this study, we presented new theory of effusive and explosive of volcanic eruptions. New explanation of eruption mechanisms was done by using the Elemental Buoyancy Theory and new K-Th-U structure of Earth, developed early by author. During investigation of effusive eruptions, it was given clear answer on the question why the light chemical elements, mainly silicon and sulfur compound, currently dominate in the volcanic ashes, gases, and in the magma lavas. At investigation of explosive mechanism, we analyzed 38 strong eruptions with Volcanic Explosivity Index (VEI) more than 4+. It was shown that there is a link between the planet configurations and volcanic eruptions. It can be found that volcano eruptions occurred at the different types of planet alignments. The phenomenon does depend neither on planet mass nor on the relative positions of planets. Also the phenomenon does not depend on the distance between planets, but often eruptions were observed when the distances between planets are multiple units. Also in work, it was demonstrated that the planet alignment affects not only natural processes on the Earth, but also impact the Sun activity. Based on the comparison phenomenon on the Earth and Sun, we get new mechanism to rapidly rising up pressure under the lithospheric planes by gravity vortexes. This gravity vortex was called as terrestrial magmatic protuberances.
\end{abstract}

\section{Keywords}

Volcanic Eruption, Effusive and Explosive Mechanisms, ${ }^{40} \mathrm{~K}$ Nuclear Thermal Layer, Terrestrial Magmatic Protuberance, Gravity Vortex, Planetary Trigger

\section{Introduction}

In this study, the new explosive and effusive mechanisms of eruptions were suggested. Both mechanisms are based on the presence of ${ }^{40} \mathrm{~K}$ nuclear thermal layer 
under the lithospheric plates at $600 \mathrm{~km}$ depth [1]. New mechanism for strong eruptions is connected with new inner structure of Earth based on the theory of buoyancy of elements and new K-Th-U structure of Earth. Criticisms of the old Earth's model and geoneutrino experiments can be found in [1] and [2]. Now we only note that the possibility of registration only fuel elements $\left({ }^{232} \mathrm{Th}\right.$ and $\left.{ }^{238} \mathrm{U}\right)$ casts doubt on advisability of carrying out the long and expensive experiments such as the KamLAND and Borexino Experiments. Thus the attempt to restore Earth's structure by using geoneutrino experiments ended unsuccessfully before beginning of these experiments.

Due to the presence of a fuel nuclear layer of ${ }^{40} \mathrm{~K}$, which is allocated at depths of $\sim 600 \mathrm{~km}$, on boundary between upper and low mantle, the earlier abstract theories of subduction and continental drift obtained understandable and obvious physical meaning. However the author believes that presence of ${ }^{40} \mathrm{~K}$, which it is possible to name figuratively as "thermal nuclear bomb" or "hellish pan" is as much surprise for most volcanologists. The current volcanology for effusive eruptions, which is based on the subduction of lithospheric plates, must be revised right after revising the subduction theory and theory of continents drifts. Note that it is a fundamentally new geophysics and new volcanology.

This ${ }^{40} \mathrm{~K}$ fuel layer is the basis of new volcanology and seismology theory, subduction and continental drift. Highlights that the geoneutrino teams did not recognize their mistakes, more than these losers do not nominate the author on the Nobel Prize for discovery of the role of ${ }^{40} \mathrm{~K}$. This great discovery is made so casually with the help of the Archimedes buoyant theory and periodic table of elements. In this situation author strongly recommends visit Turkish public baths, and in them remember about Eureka and Archimedes buoyant law. Based on buoyant theory and presence and ${ }^{40} \mathrm{~K}$ hot fuel layer a new theory of effusive mechanism of eruptions is developed.

However, the author believes that not everyone has realized and understood how great discoveries are made. Therefore, the author recommends recalling the other great discovery of the Archimedes' lever. In Section 4 a new theory of explosive mechanism of eruptions also is suggested and it is investigated a correlation between explosive eruptions and planetary configurations. However in this work it was supposed that planet configuration is the reason of activation the ${ }^{40} \mathrm{~K}$ nuclear layer and of rapid increasing of pressure in the magmatic layers under lithospheric plates which lead to explosive eruptions.

The purpose of this study is to find out how different activation mechanisms of the ${ }^{40} \mathrm{~K}$ thermal nuclear layer, located at a depth of $660 \mathrm{~km}$, lead to different types of volcanic eruptions. The results of the study have a fundamental value for geosciences, and purely practical value for volcanology, seismology and climatology.

\section{Materials and Methods}

\subsection{Volcanic Eruptions as Studying Object}

It is well known that the intensity of volcanic eruptions is well described by Vol- 
canic Explosivity Index (VEI) [3]. In this study, the 38 volcanic eruptions (1600-2020), with VEI more than 4, were object of our investigation. Note that the eruption with VEI $>4$ corresponds to tephra volume with size more $0.1 \mathrm{~km}^{3}$, cloud volcanic column height is more than $10 \mathrm{~km}$ a.s.l. and continuous blast is during more than 12 hours. Below these eruptions of volcanoes were designated as V4+. The volcanic data were obtained from Siebert et al., [4] or datasets of NGDC/WDS Global Significant Volcanic Eruptions Database [5] and "Smithsonian National Museum of Natural History” [6] and Global Volcanism Program [7].

The investigations of apparently effusive, explosive and hybrid explosive-effusive activities were presented in the several works, e.g., [8] [9] [10] and many others. The details of previous explosive and effusive models could be found in review Cassidy et al., [11] and references therein.

\subsection{Elemental Buoyancy Theory and the Geothermal Processes}

A multi-layered model of the Earth's structure representing the K-Th-U terrestrial reactor has been described in detail in [1] and [2]. This model schematically was presented in Figure 1. The model is based on the buoyancy theory of chemical elements. Light chemical elements and isotopes in the melts float up, and heavy ones sink down.

Note that in astrophysics the analogue of the elemental buoyancy theory is well known; the corresponding astrophysical model has received the name an

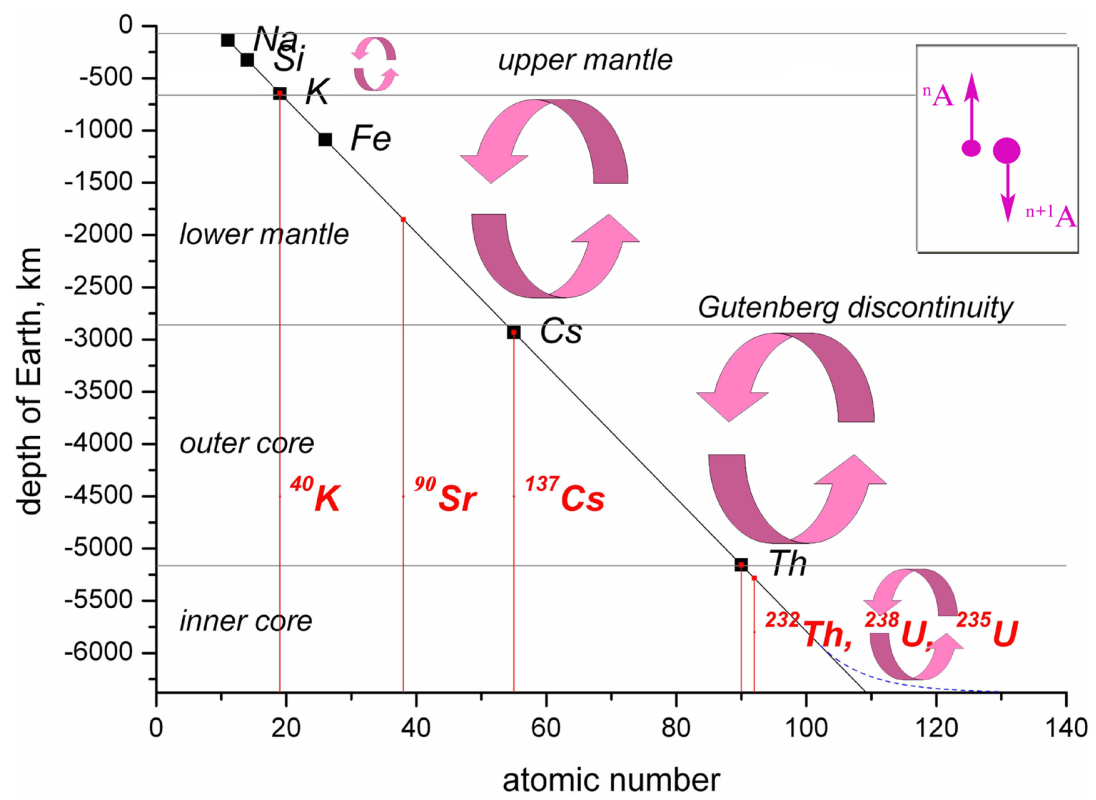

Figure 1. The scheme of terrestrial nuclear multilayer K-Th-U reactor based on isotope buoyancy theory is presented. The red lines show the basic fuel elements, such as ${ }^{40} \mathrm{~K}$, ${ }^{232} \mathrm{Th},{ }^{235} \mathrm{U},{ }^{238} \mathrm{U}$ and major products of decay such as ${ }^{137} \mathrm{Cs}$ and ${ }^{90} \mathrm{Sr}$. The red circular arrows show the shallow convection processes inside the Earth. The Sr decay level is degenerated in the "cold" planet. On plate: the buoyancy theory principal: the heavy element ${ }^{\mathrm{n}+1} \mathrm{~A}$ sinks down; the light element ${ }^{\mathrm{n}} \mathrm{A}$ floats up. 
"onion" model. However there are a number of essential differences, namely "onion" model is applied only to massive stars with mass more than 8 - 10 mass of the Sun $\left(\mathrm{M}>\mathrm{M}_{\text {solar }}\right)$. Besides in the "onion" model the potassium layer is absent due to this elements cannot be synthesized during explosion of massive stars. Note, that in metallurgy the elemental buoyancy method is using for separation of ores.

Further, due to the fact that isotopes have different masses, elemental buoyancy theory was generalized to isotopic buoyancy theory. Remind that in nuclear science the similar method is earlier repeatedly applied to isotopes separation. However in nuclear science for acceleration of separation process the terrestrial gravity has been replaced by centrifugal force of a rotating drum of a centrifuge. Thus in [1] and [2] it is offered for the first time to apply the isotopic buoyancy theory to magmatic flux of the Earth.

From buoyancy, it follows that the boundary between the upper and lower mantle is represented by ${ }^{40} \mathrm{~K}$ hot nuclear layer which allocated at a depth of 660 $\mathrm{km}$. The state of this hot fuel nuclear layer determines the seismic activity and, accordingly, it give major contribution to behavior of volcanic eruption. The thermal emissions of ${ }^{40} \mathrm{~K}$ fuel element are equal to $2.92 \times 10^{-5} \mathrm{~W} / \mathrm{kg}$ and this fuel element has a half-life period of $1.25 \mathrm{Ga}$ years.

\subsection{Historical Remarks}

In this study some critical remarks about history of seismology are highlighted. The up-to-date geophysicist declines the law of buoyancy Archimedes. Undoubtedly such position should cause serious fears. For those who did not know or prefer to forget, we remind the following.

Note that the ancient seismology exists long before Archimedes, and on Archimedes works it did not come to an end. The ancient seismological (seismoacoustic) stations were usually allocated at temples. Thus, the black date for ancient seismology and volcanology is the moment when the heathen temples were closed by the Roman Emperor Constantine I (272-337 AD). Since the temples on the remote provinces of the Roman Empire were the last to be closed, then with a high degree of probability it is possible to assert that the seismological station at the Temple of Plutonium in Hierapolis, south-western Anatolia was one of the last to be closed. The closure of this last seismic station took place in about 333 AD. A geological fault passed directly under this Temple. The fault is active to the present and the composition of volcanic gases is emitted through a seismological tunnel (called as Pluto's Gate). Thus, the system of ancient seismic stations has existed in the Mediterranean during 550 years after Archimedes, which lived in 287-212 BC.

On the other hand, we remember about the school of Pythagoras (570-490 BC) at the Temple of the Muses. This school was allocated in Crotone, in southern Italy. The Temple of the Muses was build above the geological crack and this temple also has a seismic tunnel. As it is known from historical sources two 
scholars have rescued in this shaft at the attack to school of Pythagoras. Therefore the system of seismic stations has existed in the Mediterranean at least during 200 years before Archimedes.

The selectivity of knowledge of modern geophysicists, and their reluctance to recognize the works of ancient European philosophies such as Pythagoras, Plato, Aristotle, and Archimedes look the highly suspicious.

\subsection{Astronomical Data and Method}

The history of studies about idea, that other planets are controlling volcanism on the Earth, is known for a long time. Our opponents extremely unfriendly concern to the publication such facts; though they sometimes also remember about Pliny Eld. So we specially took out the descriptions of the various points of views and references in the Section Discussion.

First of all in this study we present the facts about correlation between planet position and explosive eruptions. Each reader can give own interpretation to these facts. To investigate the causes of explosive eruptions, we need to measure astronomical angles and distances between planets. In this work the Orbit Viewer java applet from Osamu and Ron [12], which was created by Osamu Ajiki (AstroArts Inc.) in 1996 and modified by Ron Baalke (NASA/Jet Propulsion Laboratory, below JPL) in 2000-2001, has been used. The original Orbit Viewer is an interactive applet that displays the orbit of the small bodies such as comets and asteroids in the solar system in $3 \mathrm{D}$ projection. The orbits may be shown forwards or backwards. For example, 1P/Halley comet visualization was presented at JPL Small-Body Database Browser [13]. For simplicity, all figures below are presented in $2 \mathrm{D}$ projection in the planetary plane. In this projection, the direction of planet rotation is counter clockwise. The applet Orbit Viewer has been adapted to measure the angles between the planets of the solar system and to find the proportions in the planet positions. In this study the following abbreviations were used: Mercury-Mr, Venus-V, Earth-E, Mars-M, Jupiter-J, Saturn-S, Uranus-U, Neptune-N.

The planets, which are located along the line, are marked as Planet $_{1}-$ Planet $_{2}-$ Planet $_{3}$, in the order of the distance from the Sun. The calculation of the angle (in degree) between the three planets was made when the vertex of an angle is placed on the more distant planet. For example, the angle for the linearity of $\mathrm{J}-\mathrm{E}-\mathrm{V}$ is the smaller angle between the two lines of JE and JV. The ratio of the distances between the planets was designated as Planet ${ }_{1}$-Planet ${ }_{2} /$ Planet $_{3}$-Planet ${ }_{4}$. For example, the ratio of the distance between Saturn and Earth to the distance between Earth and Venus was denoted as SE/EV.

During our investigation of planetary geometry the alignment angle between lines Planet $_{i}-$ Planet $_{j}$ and Planet $_{j}-$ Planet $_{k}$ were used. This alignment angle $\alpha_{i j k}$ was defined in degree as next:

$$
\alpha_{i j k}=180^{\circ} / \pi \cdot \arccos \left(\left(R_{i j}^{2}+R_{j k}^{2}-R_{i k}^{2}\right) /\left(2 \cdot R_{i j} \cdot R_{j k}\right)\right)
$$

where $i, j, k$-tree planets in the Solar System; $x_{i}, y_{i}, z_{i}$-coordinates $i$-planet 
and distance between $i$ and $j$ planets is equal to

$R_{i j}=\left(\left(x_{i}-x_{j}\right)+\left(y_{i}-y_{j}\right)+\left(z_{i}-z_{j}\right)\right)^{1 / 2}$.

Due to our search for tracer of interference, the spatial ratio designations as a ratio of distances between planets were used. This ratio was defined as:

$$
N_{i j}=R_{j 3} / R_{i 3}
$$

where $R_{i, j 3}$-distance between $i, j$ planets to Earth and $R_{j 3}>R_{i 3}$. We are looking for the spatial ratio close to integer values.

\section{New Effusive Mechanism}

In [1] and [2] it was shown that the ${ }^{40} \mathrm{~K}$ fuel layer is allocated on the border of upper mantle and low mantle disarranges and revisions several up-to-date geosciences theories. This hot fuel layer is a part of the multi layers K-Cs-Th-U terrestrial reactor, see Figure 1.

This ${ }^{40} \mathrm{~K}$ layer is presented in the enlarged scheme of effusive mechanism of volcanic eruption is draw in Figure 2. The labels in Figure 2(a) with numbers (1), (2), and (3) are corresponded to the plate shift, subduction and effusive mechanism of eruption. The buoyancy theory and separation process of elements and isotopes were presented in right site of Figure 2(a) by label number (4). As it can be seen from Figure 2(a), a piece of the lithospheric plate (1) slowly sinks into the magma and when it reaches the hot ${ }^{40} \mathrm{~K}$ nuclear layer, it begins to melt. The melting products float up and form a mountain range and network of erupting volcanoes (3). This process is slow and gradual, and it mainly corresponds to effusive mechanism of eruptions. Fundamentally new in this description is the presence of heated nuclear layer. Now we will consider in more details, what processes the presence of $\mathrm{a}^{40} \mathrm{~K}$ layer will lead to.

As it is known, the chemical composition of volcanic gases is mainly determined by water vapour, $\mathrm{CO}_{2}, \mathrm{CO}, \mathrm{N}_{2}, \mathrm{SO}_{2}, \mathrm{SO}, \mathrm{S}_{2}, \mathrm{H}_{2}, \mathrm{NH}_{3}, \mathrm{HCl}, \mathrm{HF}, \mathrm{H}_{2} \mathrm{~S}, \mathrm{CH}_{4}$, $\mathrm{H}_{3} \mathrm{BO}_{3}, \mathrm{Cl}$ and $\mathrm{Ar}$, that is, it consists of the chemical elements presented in the upper part of the periodic table (Figure 2(b)). This fact can be quite simply explained by the fact that the hot nuclear layer forms a thermocline (thermopause), which on the one hand prevents global convective processes, and on the other separates the elements located in the periodic table above and below the ${ }^{40} \mathrm{~K}$ fuel layer. Light elements, such as $\mathrm{Mg}, \mathrm{Al}, \mathrm{Si}$ and $\mathrm{S}$, will float up, and elements heavier than K, such as Fe and Ni, will sink down, Figure 2(a). Thus, in volcanic ashes, gases and lavas, sulphur compounds are found in large quantities, but elements, such Ag, Au, Pt or more heavy Th and U, are extremely rarely found, due to they allocated deeply into the Earth.

Further, it is interesting to note that chemical compounds such as FeS or $\mathrm{Ni}_{2} \mathrm{Si}$, consisting of chemical elements located above and below potassium, will dissociate into atoms in the hot layer: some of which will float upward, while others will sink into the interior of the planet.

$$
\begin{aligned}
& \mathrm{FeS} \rightarrow \mathrm{Fe}+\mathrm{S} \rightarrow \mathrm{Fe}_{\downarrow}+\mathrm{S}_{\uparrow} \\
& \mathrm{Ni}_{2} \mathrm{Si} \rightarrow 2 \mathrm{Ni}+\mathrm{Si} \rightarrow 2 \mathrm{Ni}_{\downarrow}+\mathrm{Si}_{\uparrow}
\end{aligned}
$$




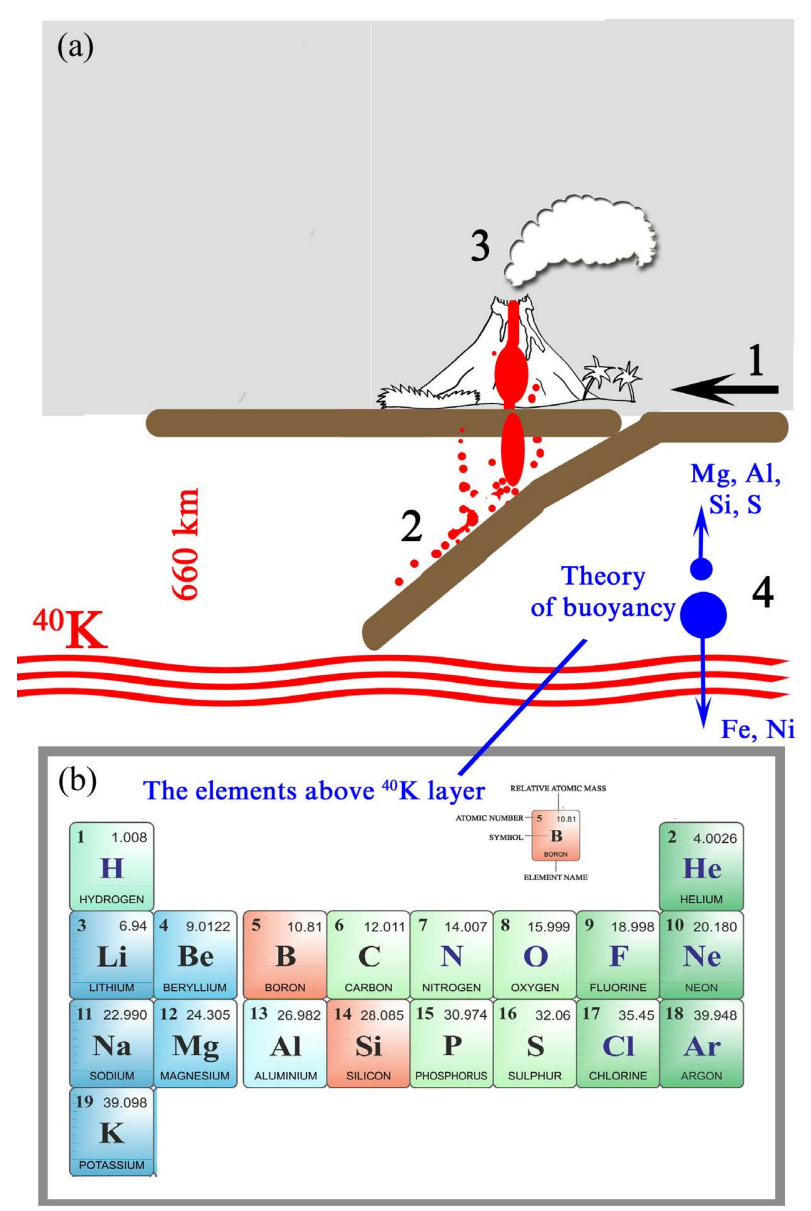

Figure 2. (a)-The scheme of effusive mechanism of volcanic eruption, in which it was demonstrated that the presence hot nuclear ${ }^{40} \mathrm{~K}$ fuel layer on the border of upper mantle and low mantle. (b) - The part of the periodic table with elements, allocated above hot fuel nuclear ${ }^{40} \mathrm{~K}$ layer. Number labels indicate: (1) - influence of a hot fuel layer on the continent drift; (2)-on the process of subduction; (3)-process of effusive volcano eruption.

Therefore, such species, as $\mathrm{FeS}$ and $\mathrm{Ni}_{2} \mathrm{Si}$, if present inside the planet, they will be in limited quantities. It can also be concluded that the widely used Bulk Silicate Earth (BSE) model is applicable only within the upper mantle, since all volume of silicium, will float upward.

In connection with the discussion of $\mathrm{FeS}$ and $\mathrm{Ni}_{2} \mathrm{Si}$, the following question arises: On what basis is it assumed that the Earth's core consists of iron? In the framework of modern geophysics, there is no answer to this question. In this question, geophysicists refer to astrophysicists, who in turn refer to specialists in reactors and accelerators. In this situation, it seems difficult to find the arguments that formed the basis of this statement. Recall that, according to modern Galactic Chemical Evolution (GCE) models, iron cannot be synthesized in the solar system, since the solar reactor is very weak, capable of synthesizing mainly hydrogen and helium [14] [15]. Iron should be synthesized on stars with a mass of 8 - 10 solar masses $\left(\mathrm{M}_{\text {star }} \sim 8-10 \mathrm{M}_{\text {solar }}\right)$. However, the transfer of large 
amounts of iron and nickel to the solar system, followed by the formation of the Earth's core, which, according to most modern geophysical theories, consists mainly of iron and nickel, is surprising.

The author with surprise finds out that the transfer equation is absent in galaxy chemical models (GCE). Note that for 80 years of the existence of GCE models, none of the astrophysicists asked a question about the transfer equation. The error is visible even in the name of the models Galactic Chemical Evolution (GCE), so these models are not Galactic Chemical Transport (GCT) models.

According to up-to-date GCE models all heavy elements including gold, platinum, and silver should be synthesized on stars having the powerful reactor, on different stars and after transferred to Earth and allocated in the oldest geological falls. Therefore an actually the theory of terrestrial ores origin is absent.

Moreover, no such elements, as iodine, cesium and uranium, were found in the spectrum of the Sun [16] [17] [18] [19], so there is a problem of "exclusive delivery" of these elements to Earth. As used here the "exclusive delivery" means that delivery of elements from other remote stellar systems (Neutron Stars, NS) was carried out passing the Sun, Venus, Mars and the Moon. Probably astrophysicists seriously believe that uranium was transported to the solar system with the help of alien spacecrafts. Also, if we say about exclusive distributed, we can suspect an alien in each pharmacist, who sold us a bottle of iodine. It is clear that such conclusion following from the astrophysical GCE models can raise a smile. On the other hand the absence of the ores origin theory as well as the water synthesis extremely adversely affects on the image of geophysics.

In this section we have considered the nuclear ${ }^{40} \mathrm{~K}$ layer activation at subduction process, by pressure of the lithospheric plate. In the following section we will consider process of activation of this layer by the planetary impact.

\section{New Explosive Mechanism}

After the text edit has been completed, the paper is ready for the template. Duplicate the template file by using the Save As command, and use the naming convention prescribed by your journal for the name of your paper. In this newly created file, highlight all of the contents and import your prepared text file. You are now ready to style your paper.

\subsection{Four Climatic Significant Explosive Eruptions}

As it is known, one of climatic significant eruptions is the 1991 eruption of the volcano of Mount Pinatubo. During this eruption, so much volcanic ash was emitted in a stratosphere that it cooled the Earth's surface for the next 3 years. Other examples are the 1980 eruption of Mount St. Helens volcano and El Chichón volcano eruptions in 1982. Data on the most famous volcanoes in climatology such as Tambora (1815), St. Helen (1980), El Chichón (1982) and Mt. Pinatubo (1991) can be found for example in [20]-[25], and in the many other references. Studying the climate changes caused by these eruptions, attention was drawn to the unusual location of the planets at the time of powerful explosive eruptions. 
Thus St. Helens and Pinatubo eruptions occurred at times when there were linear configurations of planets (Figure 3). The Saturn-Mars-Venus alignment was recorded at the St. Helen eruption (Figure 3(a)) and Jupite-Mars-VenusEarth alignment has placed at the Pinatubo eruption (Figure 3(b)). In the figures the acute angle between the directions to the planets is specified in degrees, so the angle between the straight lines Saturn-Mars and Saturn-Venus at St. Helens eruption, 1980 is equal to $0.68^{\circ}$. For convenience, in this study all figures are presented in shape of $2 \mathrm{D}$-geometry, with projection in the planetary plane. In select projection the planes rotate around Sun in CCW counter clockwise (CCW) direction.

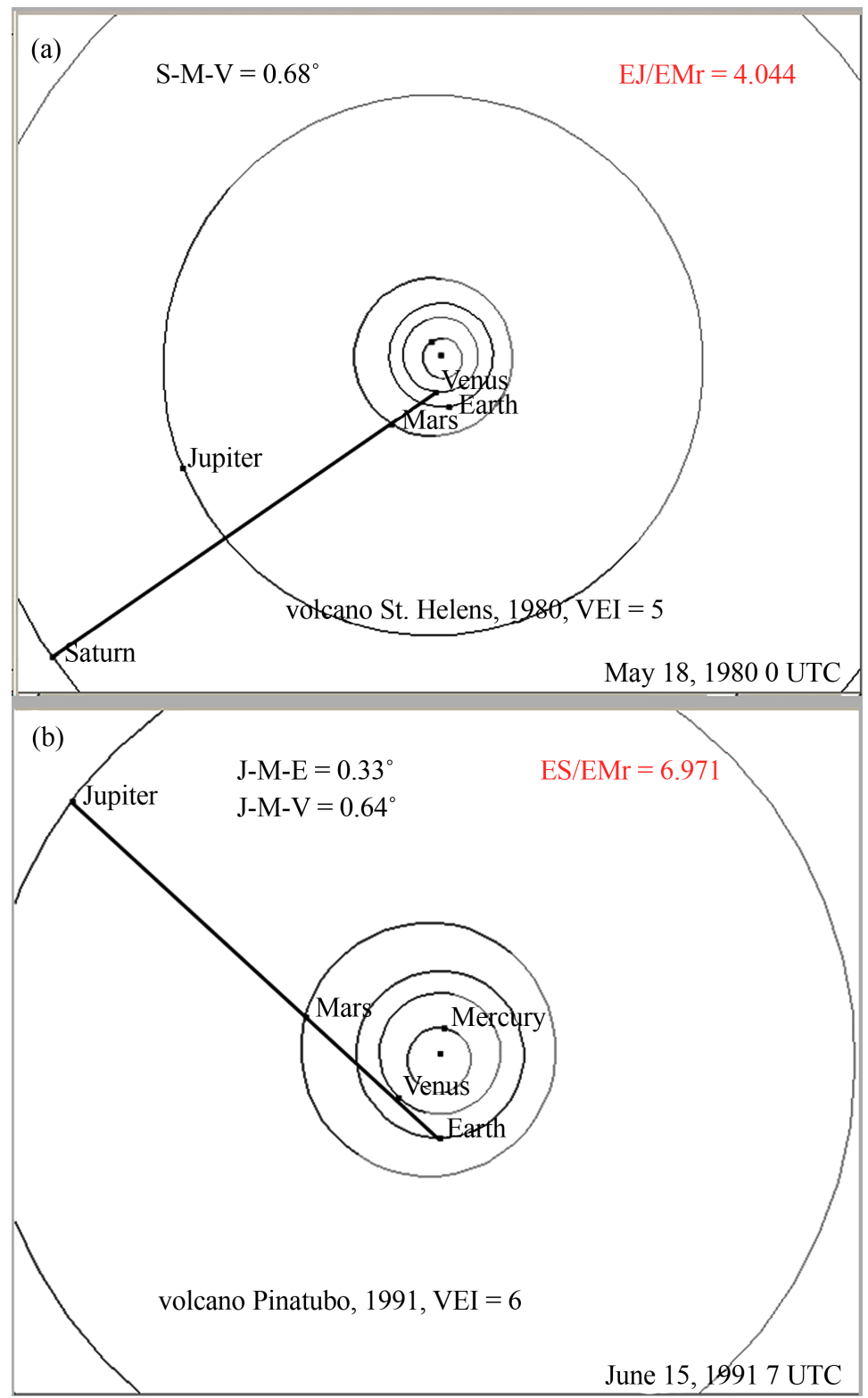

Figure 3. The climatic significant eruptions: (a)-St. Helen (1980), VEI $=5$, with the Saturn-Mars-Venus alignment; (b) -Pinatubo eruption (1991), VEI = 6, with the 4-planet Jupiter-Mars-Venus-Earth alignment. The alignment angles were presented in additional. 
Two other strong climatic significant eruptions eruption of El Chichón volcano were presented in Figure 4. The El Chichón volcano erupted in March 1982 with VEI $=4+$ at the Saturn-Mars-Earth alignment (Figure 2(a)) and later erupted in April 1982 with VEI = 5 at the Saturn-Mars-Earth and Jupiter-VenusMercury alignments.

The question is can these apparent correlations arise by chance or is there some underlying physical phenomenon that is involved? To found answer to this question we investigated major explosive eruptions with VEI $>4+$, which took place in period 1600-2020 years. However even from Figure 3 and Figure 4 it is

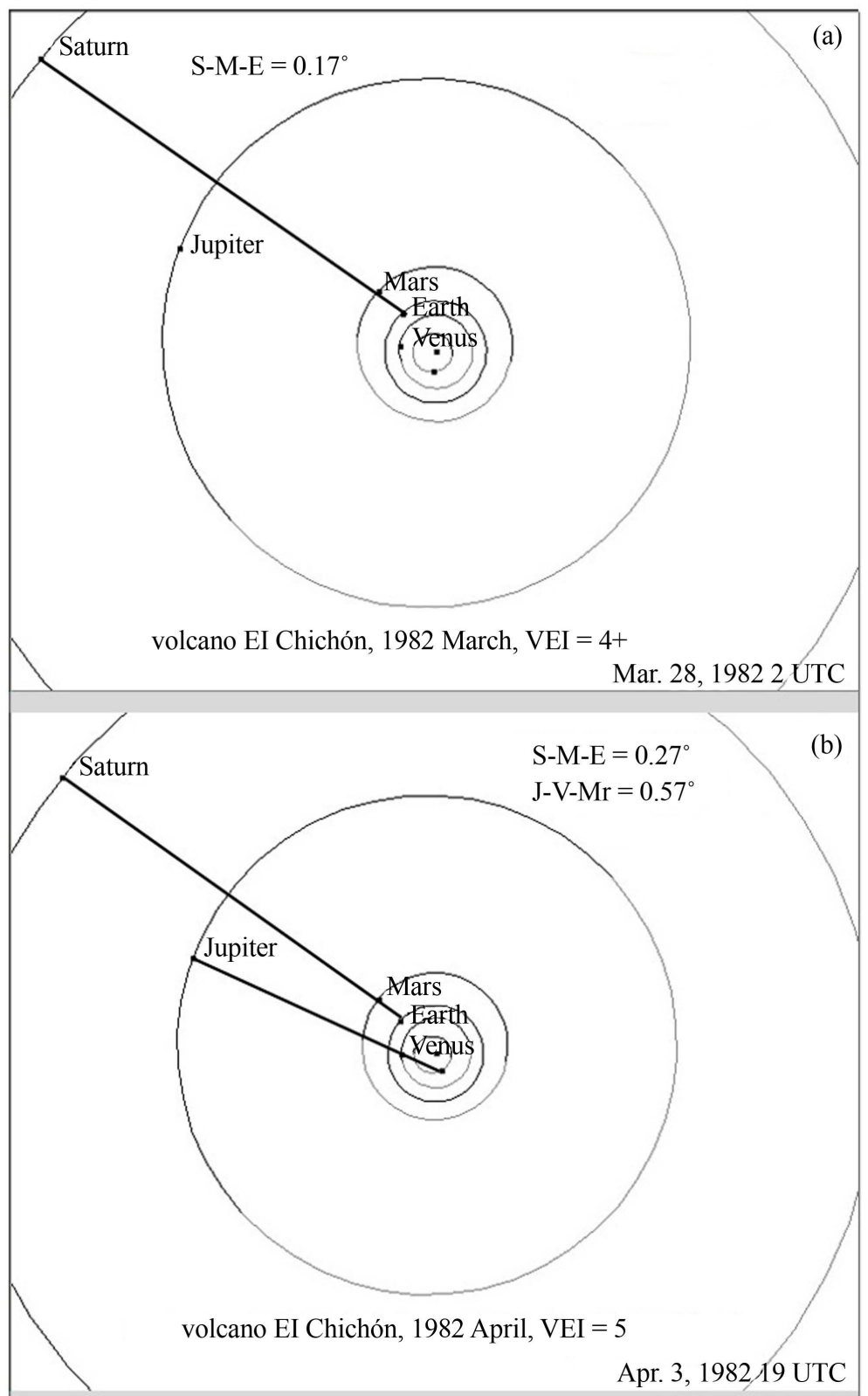

Figure 4. Two eruptions of El Chichón volcano: (a)-El Chichón eruption in March 1982, VEI $=4+$ at the Saturn-Mars-Earth alignment; (b) -El Chichón eruption in April 1982 , VEI $=5$ at the Saturn-Mars-Earth and Jupiter-Venus-Mercury alignments. 
clearly see that it is not Jupiter tidal effect. But then it is possible to come out with the assumption that at these eruptions we deal with the wave gravity process which is known as Kepler conjunction or Pythagoras-Plato waves (see Discussions).

\subsection{Planet Configurations for Major Explosive Eruptions}

Above in Section 4.1 we have considered only four cases. The size of article does not allow presented all figures of 38 strong explosive volcano eruptions, which occurred during last four hundred years. The geometry calculations of 38 eruptions have been presented below in Tables 1-3. The full set of figures of 2D planet geometry also was present in Figures S1-S19 in the Supplementary.

As it could be shown from Tables 1-3, the nature more variously and the alignment planet phenomenon is not limited by two simple schemes described in Figure 3 and Figure 4. The next types of interference schemes are presented in Table 2 and Table 3. The L-type is corresponded to the alignment, in which the Earth was involved in the alignment (such as in Figure S3(a) and Figure S3(b)). The X-type is corresponded to the alignment, in which the Earth was not involved in the alignment (such as in Figure 1(a)). The Sun-type is type in which the Sun takes place in the alignments with the planets. The multi planet type (MP) is type when the multi planets, more than 3, were involved in alignment (such as in Figure 1(b)) and the multi alignment type (MA) is when the

Table 1 . The planet alignments with angle less than $0.5^{\circ}$ for largest volcano eruptions.

\begin{tabular}{|c|c|c|c|c|c|c|c|}
\hline Number & Figures & Volcano Name & VEI & Data & Volcano Type & Alignment & Scheme \\
\hline \multicolumn{8}{|c|}{ Planets alignments } \\
\hline 1 & 1a & St. Helens & 5 & 18.05 .1980 & Stratovolcano & S-M-V & $\mathrm{X}$ \\
\hline 2 & $1 b$ & Pinatubo & 6 & 15.06.1991 & Stratovolcano & J-M-V-E & $\mathrm{L}$ \\
\hline 3 & $2 \mathrm{a}$ & Fuji & 5 & 15.12 .1707 & Stratovolcano & S-E-M & $\mathrm{L}$ \\
\hline 4 & $2 \mathrm{~b}$ & Galunggung & 5 & 08.10 .1822 & Stratovolcano & S-E-Mr & $\mathrm{L}$ \\
\hline 5 & $3 a$ & Vesuvius & 5 & 16.12 .1631 & Complex volcano & $\mathrm{E}-\mathrm{V}-\mathrm{Mr}$ & $\mathrm{L}$ \\
\hline 6 & $3 b$ & Gamkonora & 5 & 20.05 .1673 & Stratovolcano & M-V-E & $\mathrm{L}$ \\
\hline 7 & $4 \mathrm{a}$ & El Chichón & 5 & 28.03 .1982 & Tuff cone & S-M-E & $\mathrm{L}$ \\
\hline 8 & $4 \mathrm{~b}$ & El Chichón & 5 & 27.05 .1982 & Tuff cone & S-M-V & $\mathrm{L}$ \\
\hline 9 & $5 a$ & Colima & $4+$ & 20.01 .1913 & Stratovolcano & $\mathrm{J}-\mathrm{M}-\mathrm{Mr}$ & $\mathrm{X}$ \\
\hline 10 & $5 b$ & Tolbachik & $4+$ & 06.07.1975 & & $\mathrm{J}-\mathrm{M}-\mathrm{V}$ & $\mathrm{X}$ \\
\hline 11 & $6 a$ & Askja & 5 & 29.03 .1875 & Stratovolcano & $\begin{array}{l}\text { S-V-E } \\
\text { N-E-J }\end{array}$ & $\mathrm{L}$ \\
\hline 12 & $6 \mathrm{~b}$ & Bezymianny & 5 & 30.03 .1956 & Stratovolcano & $\mathrm{J}-\mathrm{M}-\mathrm{Mr}$ & $\mathrm{X}, \mathrm{G}$ \\
\hline 13 & $7 \mathrm{a}$ & Katla & 5 & 11.05 .1721 & Subglacial volcano & N-M-S & $\mathrm{X}$ \\
\hline 14 & $7 \mathrm{~b}$ & Santa Maria & 6 & 24.10 .1902 & Stratovolcano & N-J-S & $\mathrm{X}$ \\
\hline
\end{tabular}


Table 2. The planets and sun alignments for the largest volcano eruptions.

\begin{tabular}{|c|c|c|c|c|c|c|c|}
\hline Number & Figures & Volcano Name & VEI & Data & Volcano Type & Alignment & Scheme \\
\hline \multicolumn{8}{|c|}{ Planets and Sun alignments } \\
\hline 1 & $8 a$ & Shikotsu & 5 & 19.08 .1739 & Caldera & $\mathrm{J}-$ Sun-Mr & Sun \\
\hline 2 & $8 \mathrm{~b}$ & Cosiguina & 5 & 20.01 .1835 & Stratovolcano & M-Sun-Mr & Sun \\
\hline 3 & $9 \mathrm{a}$ & Huaynaputina & 6 & 19.02 .1600 & Stratovolcano & J-M-Sun & Sun \\
\hline 4 & $9 b$ & Novarupta & 6 & 6.06 .1912 & Caldera & S-V-Sun & Sun \\
\hline 5 & $10 \mathrm{a}$ & Shikotsu & 5 & 23.09 .1667 & Caldera & $\begin{array}{l}\text { G-E-Sun } \\
\text { S-Sun-M }\end{array}$ & Sun, G \\
\hline 6 & $10 \mathrm{~b}$ & Hudson, Cerro & 5 & 12.09 .1991 & Stratovolcano & $\begin{array}{l}\text { J-Mr-V-E } \\
\text { G-V-Sun }\end{array}$ & $\mathrm{L}, \mathrm{G}$ \\
\hline 7 & $11 \mathrm{a}$ & Komaga-Take & 5 & 31.07 .1640 & Stratovolcano & $\begin{array}{c}\text { J-Sun-V } \\
\text { S-M-E }\end{array}$ & Sun, L \\
\hline 8 & $11 b$ & Puyehue-Cordón Caulle & 5 & 04.06 .2011 & Stratovolcano & J-V-Mr-Sun & Sun, $\mathrm{X}$ \\
\hline 9 & $12 \mathrm{a}$ & Ksudach & 5 & 28.03 .1907 & Stratovolcano & U-V-Sun-J-N & Sun, $\mathrm{X}$ \\
\hline 10 & $12 b$ & Shiveluch & $4+$ & 12.11 .1964 & Stratovolcano & N-Sun-E-J & Sun, L \\
\hline 11 & $13 \mathrm{a}$ & Grimsvotn & $4+$ & 08.06 .1783 & & J-M-Sun & Sun \\
\hline 12 & $13 b$ & Okataina & 5 & 10.06 .1886 & Lava domes & U-J-Sun & Sun \\
\hline 13 & $14 \mathrm{a}$ & Spurr, Alaska & 4 & 17.09.1992 & Stratovolcano & J-Mr-Sun-E & Sun \\
\hline 14 & $14 \mathrm{~b}$ & Manam, Papua & 4 & 24.10 .2004 & Stratovolcano & $\mathrm{J}-\mathrm{Mr}-\mathrm{M}$ & $\mathrm{L}$ \\
\hline 15 & $15 \mathrm{a}$ & Azul Cerro & $5+$ & 10.04 .1932 & & E-Mr-Sun & Sun \\
\hline 16 & $15 b$ & Colo Una-Una, Indonesia & 4 & 23.07 .1983 & Stratovolcano & M-Sun-V & Sun \\
\hline
\end{tabular}

Table 3. Multiply alignments for largest volcano eruptions.

\begin{tabular}{|c|c|c|c|c|c|c|c|}
\hline Number & Figures & Volcano Name & VEI & Data & Volcano Type & Alignment & Scheme \\
\hline \multicolumn{8}{|c|}{ Multiply alignments } \\
\hline 1 & $16 \mathrm{a}$ & Kharimkotan & 5 & 08.01 .1933 & Stratovolcano & $\begin{array}{c}\text { J-M-E } \\
\text { V-Mr-Sun }\end{array}$ & L, Sun \\
\hline 2 & $16 \mathrm{~b}$ & El Chichón & 5 & 03.04 .1982 & Tuff cone & $\begin{array}{l}\text { S-M-E } \\
\text { J-V-Mr }\end{array}$ & $\mathrm{L}, \mathrm{X}$ \\
\hline 3 & $17 \mathrm{a}$ & Furnas & $4+$ & 03.09 .1630 & Stratovolcano & $\begin{array}{l}\text { S-M-V } \\
\text { J-E-Sun }\end{array}$ & Sun, $\mathrm{X}$ \\
\hline 4 & $17 \mathrm{~b}$ & Usu & 5 & 16.08 .1663 & Stratovolcano & $\begin{array}{c}\text { S-J-V-Sun } \\
\text { S-Mr-M }\end{array}$ & Sun, $\mathrm{X}$ \\
\hline 5 & $18 \mathrm{a}$ & Katla & 5 & 02.09 .1625 & Subglacial volcano & $\begin{array}{c}\text { J-Sun-V } \\
\text { J-E-M }\end{array}$ & Sun, L \\
\hline 6 & $18 \mathrm{~b}$ & Agung & 5 & 17.03 .1963 & Stratovolcano & $\begin{array}{c}\text { J-Sun-E } \\
\text { S-V-E }\end{array}$ & Sun, L \\
\hline 7 & $19 \mathrm{a}$ & Tambora & 7 & 10.04 .1815 & Stratovolcano & $\begin{array}{c}\text { S-M-E } \\
\text { M-Sun-V }\end{array}$ & Sun, L \\
\hline 8 & $19 b$ & Krakatau & 6 & 27.08 .1883 & Caldera & $\begin{array}{c}\mathrm{J}-\mathrm{V}-\mathrm{Mr} \\
\text { M-Sun-Mr }\end{array}$ & Sun, $\mathrm{X}$ \\
\hline
\end{tabular}


multi alignments took place at the volcano eruption (such as in Figure 2(b)). The G-type is type when the Galaxy is probably involved in the alignment. Last type is more typical for earthquakes, than for strong eruptions. However the results, about correlation between this G-type and strong earthquakes, is out of scope of this work, therefore now we only shortly announced our results of such studies. Difference between L-type and X-type is shown in Figure 5.

From Tables 1-3, it could make conclusion that the effect does not depend on the size of the planets; it is observed both for minor planets such as Mercury and when the planets aligned with the Sun. Further, we note that the effect does not depend on their relative arrangement of the planets, at the same time it is uniquely determined by the geometry of the planets' locations. Based on this, it is possible to assume that the effect has an inertial nature.

The result of statistic analysis was demonstrated below in Table 4 and Table

5. The account of astronomic objects, involved in the planet alignments, was

Table 4. Account of astronomic objects, involved in the volcanic planet alignments.

\begin{tabular}{ccc}
\hline \multirow{2}{*}{ Object $^{\mathrm{a}}$} & \multicolumn{2}{c}{ Account of events } \\
\cline { 2 - 3 } & pcs. & $\%$ \\
\hline Sun & 23 & 14.7 \\
Mercury & 15 & 9.6 \\
Venus & 22 & 14.0 \\
Earth & 22 & 14.0 \\
Mars & 25 & 15.9 \\
Jupiter & 24 & 15.3 \\
Saturn & 17 & 10.8 \\
Uranus & 2 & 1.3 \\
Neptune & 5 & 3.2 \\
Galaxy & 2 & 1.3 \\
\hline
\end{tabular}

a. There are 38 studying cases of volcanic eruption with VEI $>4+$.

Table 5. Amount of interference types, involved in the volcanic planet alignments.

\begin{tabular}{cccc}
\hline \multirow{2}{*}{ Type Label } & Astronomical Objects $^{\mathrm{a}}$ & \multicolumn{2}{c}{ Account of Events } \\
\cline { 3 - 4 } & & pcs. & $\%$ \\
\hline Sun & Sun and planets & 21 & 30.4 \\
L & Earth and planets & 17 & 24.6 \\
X & planets without Earth and Sun & 12 & 17.4 \\
MA & multi alignments & 10 & 14.5 \\
MP & multi planets alignments (objects $>3)$ & 7 & 10.1 \\
G & planets and Galaxy & 2 & 2.9 \\
\hline
\end{tabular}

a. There are 38 studying cases of volcanic eruption with VEI $>4+$. 


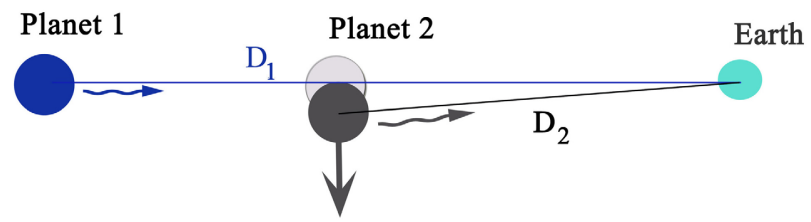

(a)

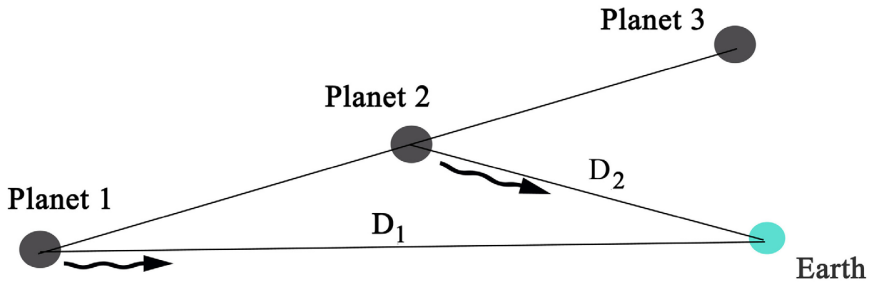

(b)

Figure 5. Two interference $\mathrm{L}$ and $\mathrm{X}$ schemes were schematically presented. (a) - Linear scheme (L), also called as Archimedes lever; (b)-triangular interference scheme (X). In both cases the gravitational waves (vortexes) were created by the planet alignment. The interference maximum would be expected when the distances between planets will be multiple.

presented in Table 4. The Sun, Venus, Earth, Mars and Jupiter participate in $14 \%-15 \%$ from the total account of studying alignments. The statistic of interference type was presented in Table 5. Note that multi alignment Type (MA) was presented in $\sim 14.5 \%$. The L-type and Sun-type are dominated at explosive volcanic eruptions.

However the next questions are of interest: How the astronomical events such as alignment of planets along a line, can influence on the other processes in solar system?

\section{Some Solar Super Storms}

In this Section it was shown that alignment interference has common nature and affects not only to Earth but also could affects to Sun. It was demonstrated on the example of two most powerful solar super storms. Another reason for considering solar storms is that the vortexes are clearly visible in the perturbations.

On July 23, 2012, at about $0208 \pm 2$ minutes UTC, the level solar activity suddenly increased and the Sun sent an unusually strong interplanetary coronal mass ejection [26] [27] [28]. This fact of the solar eruptions was well recorded by STEREO A and B satellites [29]. During the time of the solar eruptions at 2012 July 23, STEREO A and B were $121.3^{\circ}$ west and $114.8^{\circ}$ east of the Earth, at a distance of $0.96 \mathrm{AU}$ and $1.02 \mathrm{AU}$ from the Sun, respectively. The extremely high solar wind speed of $2246 \pm 110 \mathrm{~km} \cdot \mathrm{s}^{-1}$ and an unusually strong ejecta magnetic field of $109 \pm$ $1 \mathrm{nT}$ were observed near 1 AU by STEREO A [27] and in Figure 6(b). The planet alignment for the Solar Super Storm at July 23, 2012 was shown in Figure 6(a).

At 6 September 2017, 12 UTC the strong Sun flare was happened, which correlated to the X9.3 class. The SOHO satellite image (image number \#PIA21949) 

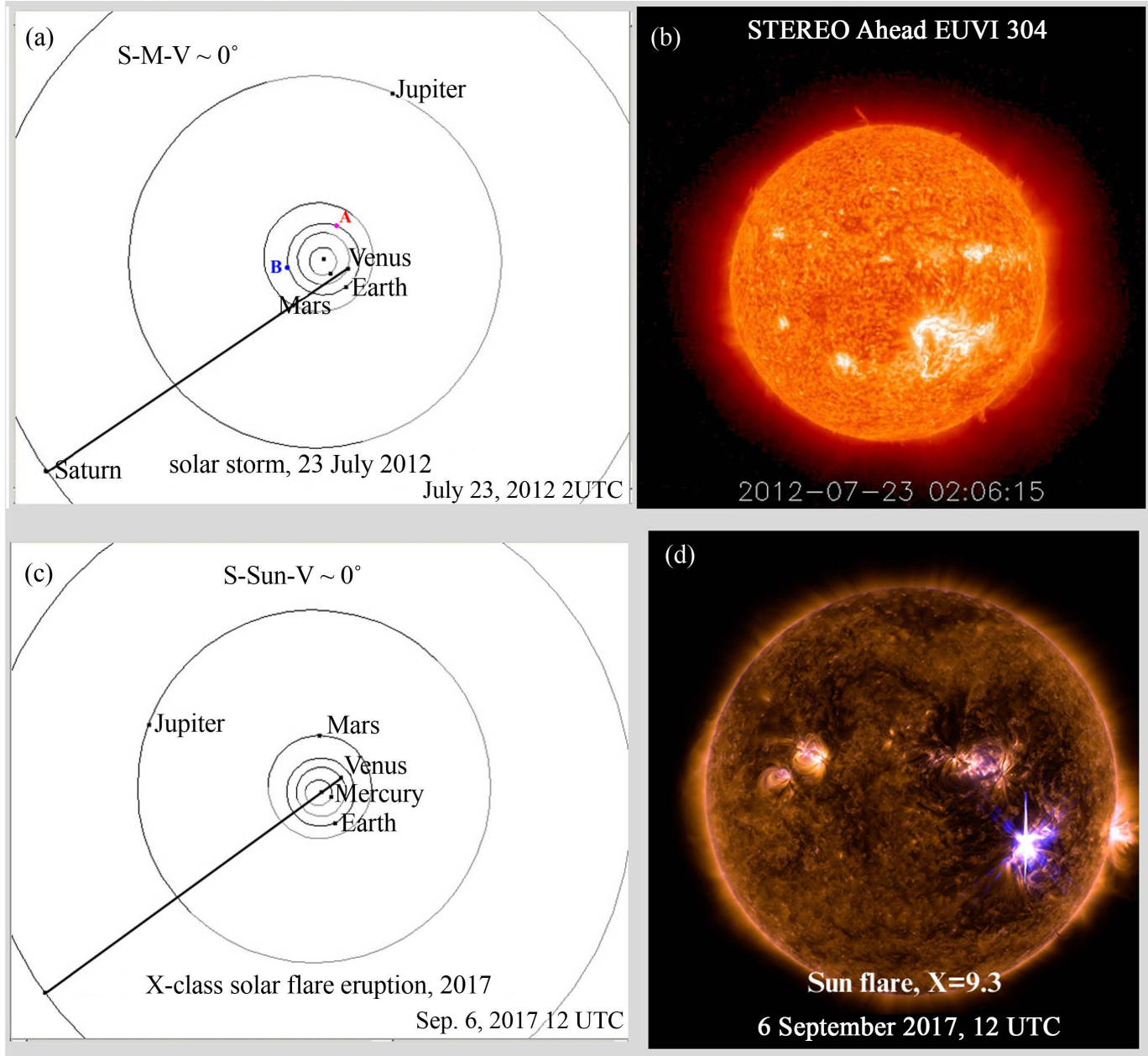

Figure 6. (a)-The Saturn-Mars-Venus planet alignment for the solar super storm at 23 July 2012, 2 UTC. The STEREO A and B satellite positions are shown as A an B symbols; (b)-the solar super image 23.07.2012 which was obtained by STEREO A; (c) - Saturn-Sun-Venus planet alignment for the strong X9.3 class Sun flare at 6 September 2017, 12 UTC; (d)_SOHO satellite image (PIA21949) at 6 September 2017, 12 UTC.

of this strong Sun flare was presented on Figure 6(d). The planet alignment at this moment at September 6, 201712 UTC was Saturn-Sun-Venus, see Figure $6(c)$.

Summarizing previous two Sections we could write next.

First, we show that the explosive terrestrial volcano eruptions and the solar protuberances have same physical nature. Secondary, previously nobody has paid attention that the subduction can partially explain only the sluggish ongoing continuous eruptions, but it does not fit to explanation of explosive volcanic processes. Note that the subduction occurs continuously, but very slowly. Thirdly, the vortex structures are clearly visible in the solar protuberances. Therefore it is possible to come out with the assumption that explosive eruptions probably took place due to suddenly rising up pressure after generation terrestrial protuberances under the lithospheric planes. The process of explosive eruptions, which we concretize below, is schematically illustrated in Figure 7. 


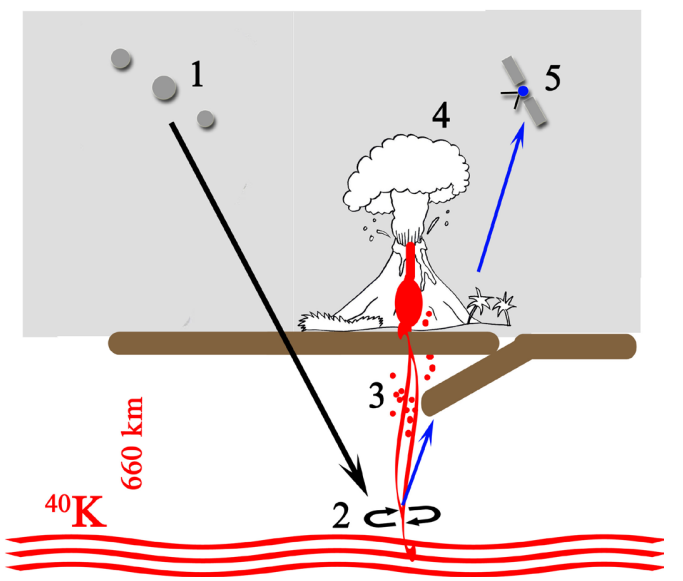

Figure 7. The scheme of explosive mechanism of volcanic eruption. Number labels indicate: (1)-astronomical event; (2) - gravity vortex; (3)-terrestrial protuberance; (4)process of explosive volcano eruption; (5)-satellite, which recorded the high-frequency signal from the magma vortex.

\section{Discussions}

In this study new explosive mechanism of volcano eruptions at the planet alignments was discovered. However such a mechanism can be called as "new" with a big stretch, since similar statements was known for a long time. In this situation we look back in the history of astronomy and below wrote some words about Archimedes' lever and Kepler conjunctions.

In the end of Bronze Age alignment phenomenon was called as Orion belt principal. Thus the astronomer Moses who lived in Minor Asia in the 12 century BC give next trick definition of Orion's belt principal, see Bible's Book of Job 38:31:

"Can you bind the chains of the Pleiades? Can you loosen Orion's belt?" (4)

In Ancient Europe this phenomenon was known since Thales of Miletus and Pythagoras as a Theory of Music. Thus we highlighted that the Pythagoras-Plato gravitational waves and its interferences were known in Europe since the times of Thales of Miletus and Pythagoras. Later Aristotle mentioned in passing about relation between seismic activity and cosmos. In the Roman Empire the alignment phenomenon was called as Archimedes' lever principal, e.g. [30] [31]. The Archimedes lever, are correlated to linear scheme (L-type), which was presented in Figure 5(a). According to Pappus of Alexandria, Archimedes' levers mind next, (Archimedes, Syracuse, 287-212 BC):

"Give me a place to stand and with a lever I will move the whole world" (5)

If to move further on a historical scale we will remember that Neo-Platonism conflict led to dramatic events. As it is know, Hypatia, the daughter of the famous mathematician and astronomer Theon, lived in Alexandria in 350/370-415 $\mathrm{AD}$. According to historical records, she taught mathematics and astronomy in the Neo-Platonism academy. One of her students failed to pass the geometry exam, became angry with Hypatia, killed her, and set fire to the world-famous 
library in Alexandria.

Remembering Hypatia, famous for her treatises on Neo-Platonism, we recall the following. There is an amazing feature in Greek philosophy, and this focus is that Greek philosophy, unlike the philosophy of the East, in particular from the Confucius doctrines, does not teach us wisdom, that is, how to live correctly, but teach us how to doubt the truth of one's knowledge properly. Thus the conflict between followers and opponents of alignment phenomenon is extending more than several thousand years; from time to time it calm down and flush up again.

In 1606 Kepler investigated the planet alignment phenomenon. Note that after Kepler [32] the author is not first who recently try to investigate this question. Early, like this in 1971 Gribben in [33] suggested that earthquakes were correlated with sunspots or planetary alignments. Later Gribben in [34] also claimed that the level of solar cycle activity is influenced by the alignments of the planets, through tidal interactions with the Sun. The effect of planet alignments on the earthquakes and sunspots was called as "Jupiter effect", a name coined for the heliocentric alignment of all the planets on the same side of the Sun [35]. Several criticisms on "Jupiter effect" were published by Meeus, Ip and Hughes [36] [37] [38] [39], with replies of Gribben and Plagemann [40] [41] [42].

In 1997 Geller wrote a critical review about earthquake prediction [43] [44]. Further in many studies the attempts were performed to find a way out of the crisis. However, it is necessary to notice that mostly researches again wrote about tidal effect at planet alignment: see [45]-[57], and references in them. The geophysicist's community conclusion on planetary-solar-terrestrial interaction was summarized in Mörner et al. in [58]. Thus the J. Kepler work [32] is not forgotten.

Therefore the crisis which has long history at present also parallel slowly ripens up inside the different branches of astrophysics. Like this in the solar activity the planetary variations are known till 1980 . However, this phenomenon is ignoring by astrophysicists. Secondary, since 2005 the astrophysicists started to ban all publications about Titius-Bode law. Although in the wave mechanics it is well known, that interference maxima are observed at integer values between interference distances. However, such "numerology" is not in honors by some astrophysicists. Note that, the generalized Titius-Bode law is useful at describe the planet formation in the Solar System, formations of Saturn's rings and spatial distributions of Jupiter, Saturn, and Uranus satellites and also is using to predict positions of Plutinoes (objects behind Pluto, in the Kuiper belt). During last years the development of new astronomical observation methods allowed determining the distribution of exoplanets nearby the remote stars. So the crisis spread to the newest exoplanets investigations.

Thirdly, in 2013 the wildest incident happened, the journal of Pattern Recognition in Physics was closed due to publication results of Kepler conjunctions. It can be argued that three well-known astronomers, namely Archimedes, Johannes Kepler and Johann Elert Bode, are fall a victim to modern "Holy Inquisition". Their sculpture and portraits and their works are presented in Figure 8. 

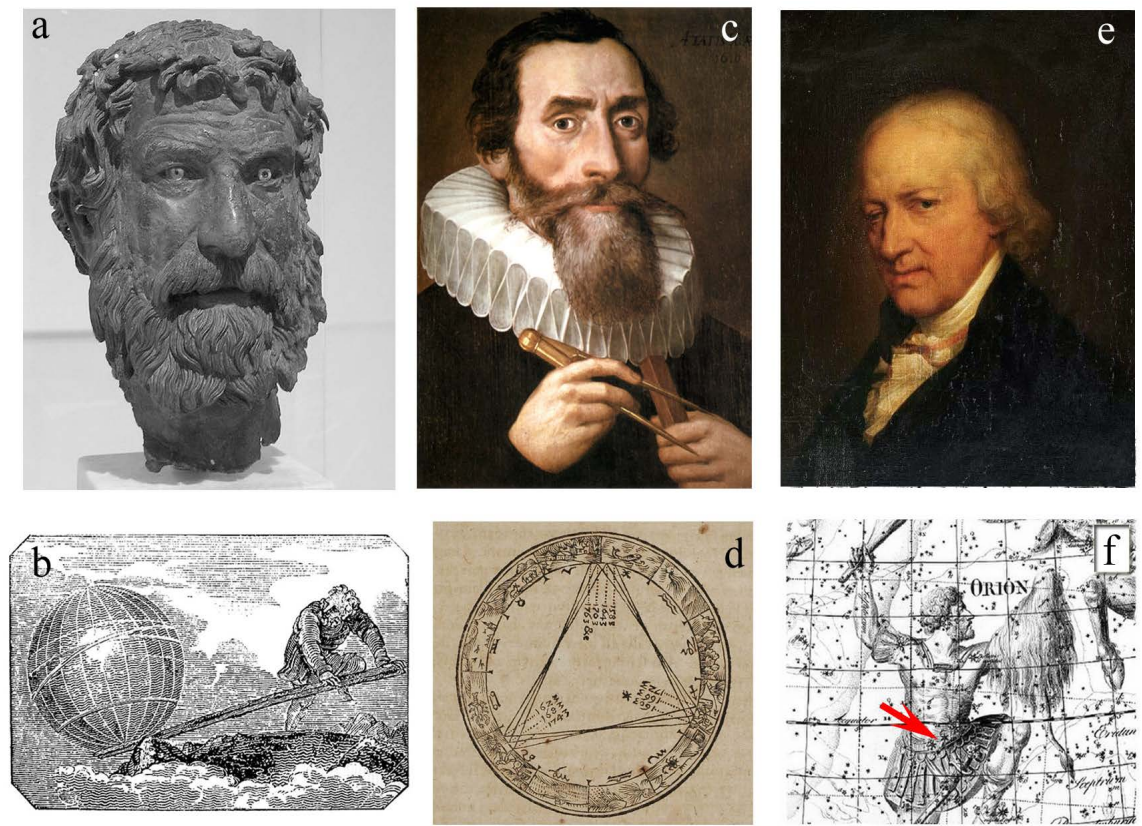

Figure 8. The well-known victims of up-to-date Holy Inquisition: (a)-Archimedes, (c)-Johannes Kepler, and (e)_Johann Elert Bode (e). In the low row the pages, illustrated their works and early discussed in the text, were shown. Labels are next: (a)-Sculpture of philosopher with a wet hair, which was lift up from Mediterranean Sea bottom together with Antikythera Mechanism. Very likely it is sculpture of Archimedes, see details in [27]; (b) - the illustration of Archimedes' levers, obtained from Mechanics Magazine published in London, 1824; (c)-portrait of Johannes Kepler by an unknown artist, 1610; (d)-The original diagram of Jupiter-Saturn conjunctions prepared by J. Kepler in [28]; (e)-portrait of Johann Elert Bode (1747-1826); (f)-illustration from Uranographia by Bode, 1801 in [55] [59]. Red arrow presents the stellar alignment in Orion belts, referenced in Bible's Book of Job 38:31.

Unfortunately, we must state that any mention of Pythagoras, Plato, Archimedes, Hypatia, and Kepler evokes violent emotions for some modern astrophysicists from panic to madness and the desire to close scientific journals and burn libraries.

\section{Conclusions}

In this study, the new mechanisms have been presented a new theory of effusive and explosive eruptions. This theory is based on the theory of the buoyancy of the elements and isotopes, and on the presence of a hot ${ }^{40} \mathrm{~K}$ fuel nuclear layer at a depth of $660 \mathrm{~km}$ on the upper-lower mantle boundary.

\section{The main results:}

\section{r1. The effusive eruptions}

The permanent, quiet burning of the ${ }^{40} \mathrm{~K}$ layer provides a simple explanation for these effusive eruptions. In work, it is specified that when pieces of the lithospheric crust are immersed in the ${ }^{40} \mathrm{~K}$ zone, they are melting with dissociation chemical compounds and next separation on heavy and light elements. Elements are heavier than potassium sink down deeply into the planet, while elements 
lighter than potassium float up. Thus, a simple explanation is given for why only light elements are mainly present in volcanic gases, ash and lavas. This is determined by the presence of a thermocline at the boundary of the upper and lower mantle and shallow convection in upper mantle. Also, this theory gives a simple explanation why volcanoes mainly allocate along subduction falls on the fixed distance from it.

\section{r2. The explosive eruptions}

It is suggested that explosive eruptions occurred at a sudden disturbance of the ${ }^{40} \mathrm{~K}$ fuel layer as a result of external influence from the solar system. The 38 explosive eruptions with VEI more than 4+ which took place between 1600 and 2020 , and also two strong solar storms were investigated. In all studying cases the explosive eruptions occurred at the planet alignments. The Sun, Venus, Earth, Mars and Jupiter participate in 14\% - 16\% from the total account of studying alignments (Table 4). Note that the L-type planet alignments, in which Earth was involved, were observed in $25 \%$ of cases (Table 5). The multi alignment type, when there are recorded more than one planet alignment, was presented in $\sim 14 \%$ of total account of studying alignments. The Sun-type alignments, in which Sun was conjoins with planets, also were recorded in $30 \%$ of total account. Thus the L-type and Sun-type alignments are dominated at the strong volcanic eruptions.

r3. In astronomy

In this work, it was shown that the planet alignment affect not only on natural processes on the Earth, but also impact on the Sun activity. Based on the comparison phenomenon on the Earth and Sun, we get new mechanism to rising up pressure under the lithospheric planes.

Further it was shown that planet alignment phenomenon does depend neither on planet mass nor from the relative positions of planets. Also this phenomenon does not depend on the distance between planets. So it is possible to come out with the assumption that phenomenon has inertial nature. Moreover the strong eruptions often were observed at the presence of integer spatial ratios of distances between planets. Thus it is possible wrote about interference effects will occur when the distances between planets are multiplied.

The creation vortex in solar protuberance gives us a slight hint that the wave processes during the alignment of the planets are associated with the exchange of gravitational spins. However it is only a guess, since we cannot visually observe the second vortex in the magma under the lithospheric plates.

\section{Acknowledgements}

Author is grateful to the Ancient Greece and Minor Asia philosophers, astronomers and mathematics for teaching how to make great discoveries.

\section{Conflicts of Interest}

The author declares no conflicts of interest regarding the publication of this paper. 


\section{References}

[1] Safronov, A.N. (2016) The Basic Principles of Creation of Habitable Planets around Stars in the Milky Way Galaxy. International Journal of Astronomy and Astrophysics, 6, 512-554. https://doi.org/10.4236/ijaa.2016.64039

[2] Safronov, A.N. (2020) A New View of the Mass Extinctions and the Worldwide Floods. International Journal of Geosciences, 11, 251-287. https://doi.org/10.4236/ijg.2020.114014

[3] Newhall, C.G. and Self, S. (1982) The Volcanic Explosivity Index (VEI) an Estimate of Explosive Magnitude for Historical Volcanism. Journal of Geophysical Research, 87, 1231. https://doi.org/10.1029/JC087iC02p01231

[4] Siebert, L., Simkin, T. and Kimberly, P. (2012) Volcanoes of the World. University of California Press, Berkeley.

[5] National Geophysical Data Center/World Data Service (NGDC/WDS) (2021) Global Significant Volcanic Eruptions Database. NOAA National Centers for Environmental Information, Asheville.

[6] Smithsonian Institution (2013) Global Volcanism Program, Volcanoes of the World, v. 4.9.4.

[7] Global Volcanism Program (2016) Global Volcanism Program, Eruptions, Earthquakes \& Emissions, V. 1.0 (Internet Application). Smithsonian Institution, Washington DC. https://volcano.si.edu/E3

[8] Castro, J., Schipper, C., Mueller, S., Militzer, A., Amigo, A., Silva Parejas, C. and Jacob, D. (2013) Storage and Eruption of Near-Liquidus Rhyolite Magma at Cordón Caulle, Chile. Bulletin of Volcanology, 75, 1-17.

https://doi.org/10.1007/s00445-013-0702-9

[9] Schipper, C.I., Castro, J.M., Tuffen, H., James, M.R. and How, P. (2013) Shallow Vent Architecture during Hybrid Explosive-Effusive Activity at Cordon Caulle (Chile 2011-2012): Evidence from Direct Observations and Pyroclastic Textures. Journal of Volcanology and Geothermal Research, 262, 25-37. https://doi.org/10.1016/j.jvolgeores.2013.06.005

[10] Botto, L., Barone, V., Canafoglia, M., Rovere, E., Violante, R., González, M., Gazzoli, D. and Schalamuk, I. (2015) Pyroclasts of the First Phases of the Explosive-Effusive PCCVC Volcanic Eruption: Physicochemical Analysis. Advances in Materials Physics and Chemistry, 5, 302-315. https://doi.org/10.4236/ampc.2015.58030

[11] Cassidy, M., Manga, M., Cashman, K. and Bachmann, O. (2018) Controls on Explosive-Effusive Volcanic Eruption Styles. Nature Communications, 9, Article No. 2839. https://doi.org/10.1038/s41467-018-05293-3

[12] Osamu, A. and Ron, B. Osamu Ajiki 1996, Ron Baalke, 2000-2001, "OrbitViewer applet", (Was Revised Autumn 2012).

http://www.astroarts.jp/products/orbitviewer/OrbitViewer-1.3.tar.gz

[13] JPL Small-Body Database Browser NASA, California Institute of Technology, Jet Propulsion Laboratory (JPL), JPL Small-Body Database Browser, Orbit Diagram, (for $1 \mathrm{P} /$ Halley).

http://ssd.jpl.nasa.gov/sbdb.cgi?ID $=$ c00001_0;orb $=1 ; \operatorname{cov}=0 ; \log =0 ; \operatorname{cad}=0 \#$ orb

[14] Burbidge, E.M., Burbidge, G.R., Fowler, W.A. and Hoyle, F. (1957) Synthesis of the Elements in Stars. Reviews of Modern Physics, 29, 547-650. https://doi.org/10.1103/RevModPhys.29.547

[15] Kobayashi, C., Karakas, A.I. and Lugaro, M. (2020) The Origin of Elements from Carbon to Uranium. The Astrophysical Journal, 900, 179. 
https://doi.org/10.3847/1538-4357/abae65

[16] Asplund, M., Grevesse, N., Sauval, A.J. and Scott, P. (2009) The Chemical Composition of the Sun. Annual Review of Astronomy and Astrophysics, 47, 481-522. https://doi.org/10.1146/annurev.astro.46.060407.145222

[17] Scott, P., Grevesse, N., Asplund, M., Sauval, A.J., Lind, K., Takeda, Y., Collet, R., Trampedach, R. and Hayek, W. (2014) The Elemental Composition of the Sun I. The Intermediate Mass Elements Na to Ca. Astronomy \& Astrophysics, 573, A25. https://doi.org/10.1051/0004-6361/201424109

[18] Scott, P., Asplund, M., Grevesse, N., Bergemann, M. and Sauval, A.J. (2014) The Elemental Composition of the Sun II. The Iron Group Elements Sc to Ni. Astronomy \& Astrophysics, 573, A26. https://doi.org/10.1051/0004-6361/201424110

[19] Grevesse, N., Scott, P., Asplund, M. and Sauval, A.J. (2015) The Elemental Composition of the Sun III. The Heavy Elements Cu to Th. Astronomy \& Astrophysics, 573, A27. https://doi.org/10.1051/0004-6361/201424111

[20] Self, S., Gertisser, R., Thordarson, T., Rampino, M. and Wolff, J. (2004) Magma Volume, Volatile Emissions, and stratospheric Aerosols from the 1815 Eruption of Tambora. Geophysical Research Letters, 31, L20608.

https://doi.org/10.1029/2004GL020925

[21] Rutherford, M.J., Sigurdsson, H., Carey, S. and Davis, A. (1985) The May 18, 1980, Eruption of Mount St. Helens: 1. Melt Composition and Experimental Phase Equilibria. Journal of Geophysical Research, 90, 2929.

https://doi.org/10.1029/JB090iB04p02929

[22] Luhr, J.F., Carmichael, I.S.E. and Varekamp, J.C. (1984) The 1982 Eruptions of El Chichón Volcano, Chiapas, Mexico: Mineralogy and Petrology of the Anhydritebearing Pumices. Journal of Volcanology and Geothermal Research, 23, 69-108. https://doi.org/10.1016/0377-0273(84)90057-X

[23] Layer, P.W., García-Palomo, A., Jones, D., Macías, J.L., Arce, J.L. and Mora, J.C. (2009) El Chichón Volcanic Complex, Chiapas, México: Stages of Evolution Based on Field Mapping and ${ }^{40} \mathrm{Ar} /{ }^{39} \mathrm{Ar}$ Geochronology. Geofísica Internacional, 48, 33-54. https://doi.org/10.22201/igeof.00167169p.2009.48.1.98

[24] Arce, J.L., Walker, J. and Keppie, J.D. (2014) Petrology of Two Contrasting Mexican Volcanoes, the Chiapanecan (El Chichón) and Central American (Tacaná) Volcanic Belts: The Result of Rift- versus Subduction-Related Volcanism. International Geology Review, 56, 501-524. https://doi.org/10.1080/00206814.2013.879375

[25] Scaillet, B. and Evans, B.W. (1999) The 15 June 1991 Eruption of Mount Pinatubo. I. Phase Equilibria and Pre-Eruption P-T- $-\mathrm{fO}_{2}-\mathrm{fH}_{2} \mathrm{O}$ Conditions of the Dacite Magma. Journal of Petrology, 40, 381-411. https://doi.org/10.1093/petroj/40.3.381

[26] Russell, C.T., Mewaldt, R.A., Luhmann, J.G., Mason, G.M., von Rosenvinge, T.T., Cohen, C.M.S., Leske, R.A., Gomez-Herrero, R., Klassen, A., Galvin, A.B. and Simunac, K.D.C. (2013) The Very Unusual Interplanetary Coronal Mass Ejection of 2012 July 23: A Blast Wave Mediated by Solar Energetic Particles. The Astrophysical Journal, 770, Article No. 38. https://doi.org/10.1088/0004-637X/770/1/38

[27] Liu, Y.D., Luhmann, J.G., Kajdič, P., Kilpua, E.K.J., Lugaz, N., Nitta, N.V., Möstl, C., Lavraud, B., Bale, S.D., Farrugia, C.J. and Galvin, A.B. (2014) Observations of an Extreme Storm in Interplanetary Space Caused by Successive Coronal Mass Ejections. Nature Communications, 5, Article No. 3481. https://doi.org/10.1038/ncomms4481

[28] Riley, P. (2012) On the Probability of Occurrence of Extreme Space Weather Events. Space Weather, 10, S02012. https://doi.org/10.1029/2011SW000734 
[29] NASA STEREO A \& B. http://stereo.gsfc.nasa.gov/instruments/instruments.shtml

[30] Safronov, A.N. (2016) Antikythera Mechanism and the Ancient World. Journal of Archaeology, 2016, Article ID: 8760513. https://doi.org/10.1155/2016/8760513

[31] Safronov, A.N. (2020) The Archimedes' Lever and Vesuvius Eruption A.D. 79. Journal of Anthropological and Archaeological Sciences, 1, 98-115. https://doi.org/10.32474/JAAS.2020.01.000119

[32] Kepler, J. (1606) De Stella Nova in Pede Serpentarii (On the New Star in Ophiuchus's Foot). Institut für Astronomie, Universität Wien, Türkenschanzstraße 17, 1180 Wien.

[33] Gribben, J. (1971) Relation of Sunspot and Earthquake Activity. Science, 173, 558. https://doi.org/10.1126/science.173.3996.558.b

[34] Gribben, J. (1973) Planetary Alignments, Solar Activity and Climatic Change. Nature, 246, 453-454. https://doi.org/10.1038/246453a0

[35] Gribben, J.R. and Plagemann, S.H. (1974) The Jupiter Effect. MacMillan, London.

[36] Ip, W.H. (1976) Chinese Records on the Correlation of Heliocentric Planetary Alignments and Earthquakes Activities. Icarus, 29, 435-436.

https://doi.org/10.1016/0019-1035(76)90144-5

[37] Meeus, J. (1975) Comments on the Jupiter Effect. Icarus, 26, 257-267. https://doi.org/10.1016/0019-1035(75)90086-X

[38] Meeus, J. (1975) Reply to Grihben and Plagemann. Icarus, 26, 270. https://doi.org/10.1016/0019-1035(75)90088-3

[39] Hughes, D.W. (1977) Planetary Alignments Don't Cause Earthquakes. Nature, 265, 13. https://doi.org/10.1038/265013a0

[40] Gribben, J. (1975) Predicting Earthquakes. Physics Today, 28, 13-15. https://doi.org/10.1063/1.2998912

[41] Gribben, J. and Plagemann, S. (1975) Response to Meeus. Icarus, 26, 268-269. https://doi.org/10.1016/0019-1035(75)90087-1

[42] Gribben, J. (1976) The Author Comments. Physics Today, 29, 11-13. https://doi.org/10.1063/1.3023411

[43] Geller, R.J. (1997) Earthquakes Cannot Be Predicted. Science, 275, 1616. https://doi.org/10.1126/science.275.5306.1616

[44] Geller, R.J. (1997) Earthquake Prediction: A Critical Review. Geophysical Journal International, 131, 425-450. https://doi.org/10.1111/j.1365-246X.1997.tb06588.x

[45] Abreu, J.A., Beer, J., Ferriz-Mas, A., McCracken, K.G. and Steinhilber, F. (2012) Is There a Planetary Influence on Solar Activity? Astronomy \& Astrophysics, 548, A88. https://doi.org/10.1051/0004-6361/201219997

[46] Bollinger, C.J. (1952) A 44.77 Year Jupiter-Venus-Earth Configuration Sun-Tide Period in Solar-Climatic Cycles. Proceedings of the Oklahoma Academy of Science, 33, 307.

[47] Condon, J.J. and Schmidt, R.R. (1975) Planetary Tides and the Sunspot Cycles. Solar Physics, 42, 529. https://doi.org/10.1007/BF00149930

[48] Hung, C.-C. (2007) Apparent Relations between Solar Activity and Solar Tides Caused by the Planets. NASA/TM-2007-214817, 1.

[49] Okhlopkov, V.P. (2014) The 11-Year Cycle of Solar Activity and Configurations of the Planets. Moscow University Physics Bulletin, 69, 257-262. https://doi.org/10.3103/S0027134914030126

[50] Scafetta, N. (2010) Empirical Evidence for a Celestial Origin of the Climate Oscilla- 
tions and Its Implications. Journal of Atmospheric and Solar-Terrestrial Physics, 72, 951-970. https://doi.org/10.1016/j.jastp.2010.04.015

[51] Scafetta, N. (2014) The Complex Planetary Synchronization Structure of the Solar System. Pattern Recognition in Physics, 2, 1-19.

https://doi.org/10.5194/prp-2-1-2014

[52] Scafetta, N. (2014) Discussion on the Spectral Coherence between Planetary, Solar and Climate Oscillations: A Reply to Some Critiques. Astrophysics and Space Science, 354, 275-299. https://doi.org/10.1007/s10509-014-2111-8

[53] Scafetta, N. and Mazzarella, A. (2015) Spectral Coherence between Climate Oscillations and the $\mathrm{M}>7$ Earthquake Historical Worldwide Record. Natural Hazards, 76, 1807-1829. https://doi.org/10.1007/s11069-014-1571-Z

[54] Stefani, F., Giesecke, A., Weber, N. and Weier, T. (2016) Synchronized Helicity Oscillations: A Link between Planetary Tides and the Solar Cycle? Solar Physics, 291, 2197-2212. https://doi.org/10.1007/s11207-016-0968-0

[55] Takahashi, K. (1968) On the Relation between the Solar Activity Cycle and the Solar Tidal Force Induced by the Planets. Solar Physics, 3, 598-602.

https://doi.org/10.1007/BF00151940

[56] Wilson, I.R.G. (2013) The Venus-Earth-Jupiter Spin-Orbit Coupling Model. Pattern Recognition in Physics, 1, 147-158. https://doi.org/10.5194/prp-1-147-2013

[57] Wood, K. (1972) Sunspots and Planets. Nature, 240, 91-93. https://doi.org/10.1038/240091a0

[58] Mörner, N.-A., Tattersall, R., Solheim, J.-E., Charvatova, I., Scafetta, N., Jelbring, H., Wilson, I.R., Salvador, R., Willson, R.C., Hejda, P. , Soon, W., Velasco Herrera, V.M., Humlum, O., Archibald, D., Yndestad, H., Easterbrook, D., Casey, J., Gregori, G. and Henriksson, G. (2013) General Conclusions Regarding the Planetary-SolarTerrestrial Interaction. Pattern Recognition in Physics, 1, 205-206. https://doi.org/10.5194/prp-1-205-2013

[59] Bode, J.E. (1801) Uranographia Sive Astrorum Descriptio.

\section{Supplementary Materials}

The supporting materials, included Figures S1-S19, could be requested from the author or obtained by Research Gate System. The planetary alignments for these Figures are described above in Tables 1-3 in the text of the manuscript. 